\title{
(4-Isopropenyl-cyclohex-1-enyl)acetic Acid Ethylester
}

\section{Soufiane EI Houssame*, Larbi EI Firdoussi and Abdellah Karim}

Laboratoire de Chimie de Coordination, Faculté des Sciences Semlalia, BP 2390 Marrakech Morocco. E-mail: soufiane@ucam.ac.ma

Received: 14 June 2001 / Accepted: 15 December 2001 / Published: 20 December 2001
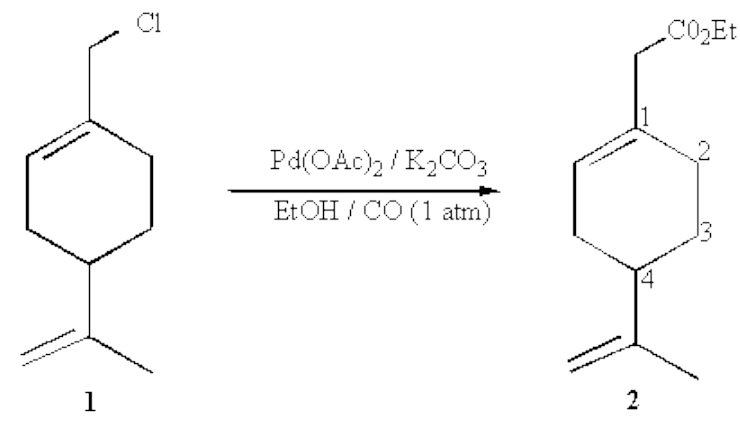

The carbonylation of allylic componds catalyzed by transition metal complexes under atmospheric pressure of $\mathrm{CO}$ is one of the most attractive tools to synthesize the $\mathrm{b}, \mathrm{g}$ - unsaturated carbonyl compounds, which are versatile building blocks [1-2].

$\mathrm{Pd}(\mathrm{OAc})_{2}(7.5 \mathrm{mg}, 0.033 \mathrm{mmol})$, anhydrous $\mathrm{K}_{2} \mathrm{CO}_{3}(135 \mathrm{mg}, 9.7 \mathrm{moml})$ and a stirring bar were placed in a three necked flask. The atmosphere was replaced with carbon monoxide and 1-Chloromethyl-4isopropenyl-cyclohexene, 1, [3] (3.3 mmol) in $5 \mathrm{ml}$ of ethanol was added under CO. The reaction mixture was stirred at $25^{\circ} \mathrm{C}$ for 1.5 hours. The reaction was followed by GC. At the end of the reaction, the mixture was filtered and the solvent was removed under vacuum. The residue was chromatographed on silica gel with Hexane / Ethyl acetate as eluent to provide the carbonylated product 2 [4] (Conversion: $100 \%$, Yield: $96 \%$ ).

$[\mathrm{a}]_{\mathrm{D}}^{20}=-43.1^{\circ}(\mathrm{c}=2.1 ; \mathrm{MeOH})$.

${ }^{1} \mathrm{H}-\mathrm{NMR}\left(300 \mathrm{MHz}, \mathrm{CDCl}_{3}\right): 5.52(1 \mathrm{H}, \mathrm{m},=\mathrm{CH}) ; 4.66\left(2 \mathrm{H}, \mathrm{m},=\mathrm{CH}_{2}\right) ; 4.1(2 \mathrm{H}, \mathrm{q}(\mathrm{J}=7.1 \mathrm{~Hz})$, $\left.\mathrm{OCH}_{2} \mathrm{CH}_{3}\right) ; 2.9\left(2 \mathrm{H}, \mathrm{S},-\mathrm{CH}_{2} \mathrm{CO}\right) ; 1.7\left(3 \mathrm{H}, \mathrm{S}, \mathrm{CH}_{3}\right) ; 1.2\left(3 \mathrm{H}, \mathrm{t}(\mathrm{J}=7.1 \mathrm{~Hz}), \mathrm{OCH}_{2} \underline{\mathrm{CH}}_{3}\right)$.

${ }^{13} \mathrm{C}-\mathrm{NMR}\left(100 \mathrm{MHz}, \mathrm{CDCl}_{3}\right): 172(\mathrm{C}=\mathrm{O}) ; 149.8(=\mathrm{C}) ; 130.9(=\mathrm{C}) ; 125.1(=\mathrm{CH}) ; 108.7\left(=\mathrm{CH}_{2}\right) ; 60.5$ $\left(\mathrm{OCH}_{2} \mathrm{CH}_{3}\right) ; 43.2\left(-\mathrm{CH}_{2}\right) ; 40.7(-\mathrm{CH}) ; 30.8\left(-\mathrm{CH}_{2}\right) ; 28.9\left(-\mathrm{CH}_{2}\right) ; 27.7\left(-\mathrm{CH}_{2}\right) ; 20.8\left(\mathrm{OCH}_{2} \underline{\mathrm{CH}}_{3}\right) ; 14.3$ $\left(\mathrm{CH}_{3}\right)$.

\section{References}

1. Tsuji, J.; Kiji, J.; Imanura, S.; Morikawa, M. J. Am. Chem. Soc. 1964, 86, 4350.

2. Kiji, J.; Okano, T.; Ono, I.; Komishi, H. J. Mol. Catal.1987, 39, 355.

3. Barllargeon, V. P.; Still, J. K. J. Am. Chem. Soc.1986, 108, 452.

4. El Houssame, S.; El Firdoussi, L.; Allaoud, S.; Karim, A.; Castanet, Y.; Mortreux, A. J. Mol. Catal. 2001, 168, 15-23.

Sample Availability: Available from the authors and from MDPI.

(C) 2001 MDPI, Basel, Switzerland. All rights reserved. 